\title{
Castleman's Disease of the Tracheal: A Case Report
}

\author{
Jing Liu ${ }^{1, a}$, Xin Liao ${ }^{1, c}$ and Wei Zhan ${ }^{2, b^{*}}$ \\ ${ }^{1}$ Department of Radiology, The Affiliated Hospital of Guizhou Medical University, Guiyang, Guizhou \\ 550004, P.R. China \\ ${ }^{2}$ Department of General surgery, The Affiliated Hospital of Guizhou Medical University, Guiyang, \\ Guizhou 550004, P.R. China

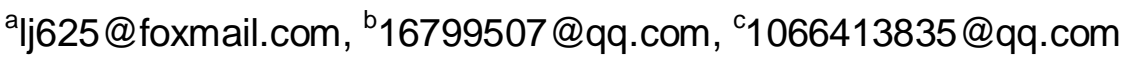

*The corresponding author

Keywords: Endotracheal; Giant lymphocyte hyperplasia; Tracheal neoplasms; Treatment evaluation; Differential diagnosis

Abstract. Castleman's disease (CD) is a chronic lymphoproliferative disorder characterized by unexplained enlarged lymph nodes. CD of the tracheal is rare, and shows unspecific manifestation with high misdiagnosis rate. Here we reported a case of tracheal CD in a 43-year-old female. Finally, the nodule of the tracheal biopsy was taken and the results confirmed CD.

\section{Introduction}

Castleman's disease (CD) is a non-caseous lymphoid proliferation, also known as angiofollicular lymphoid hyperplasia, giant lymph node hyperplasia or angiomatous lymphoid hamartoma, features of lymph node tissue and vascular proliferation. CD was firstly reported in 1956 by Benjamin Castleman [1]. It is a rare benign lymphoproliferative disorder characterized by benign lymph node hyperplasia in a single site (unicentric CD [UCD]) or in multiple sites (multicentric CD [MCD]) [2]. Patients with UCD are usually asymptomatic; however, those with MCD usually develop fever, weight loss, and peripheral lymphadenopathy.

\section{Case Presentation}

A 43-year-old woman presented dyspnea about 6 months. Physical examination: the trachea is in the middle, the thoracic is symmetrical and without abnormal, the both respiratory motion is unanimous, the intercostal spaceis not broadened, breath sounds clear. She had no peripheral lymphadenopathy, and no pertinent family history. Standard laboratory examination results were normal. The concentrations of most tumor markers were normal.
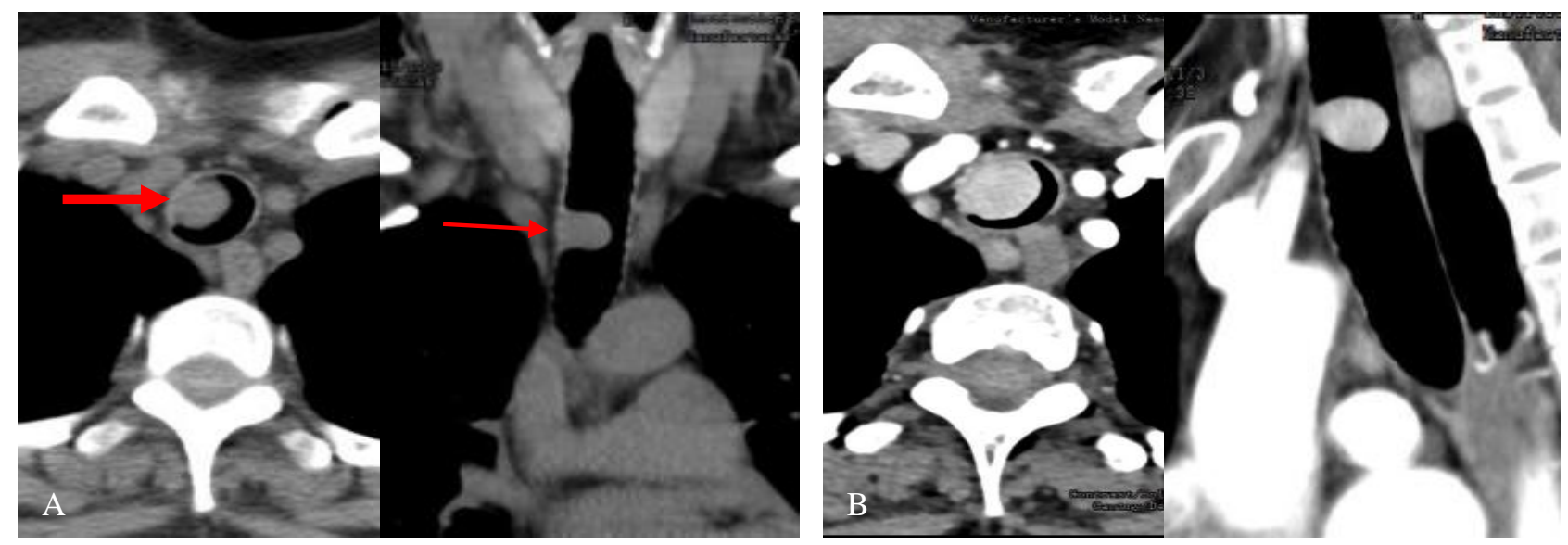

Figure 1. Chest CT

(A) Transverse and coronal CT scan. (B) Transverse and sagittal of CT enhanced scan. 
Chest CT was reviewed which was nodal-form shadow with soft tissue density in trachea about $1.1 \mathrm{~cm} \times 1.5 \mathrm{~cm}$, the edge was smooth, clean, and seen to be thickening near the walls of trachea, but the lymph nodes were enlarged in upper mediastinal. The enhanced CT scanning showied that there was a lesion in the tracheal enhanced strongly, and upper mediastinal lymph nodes were enlarged, but no hilar lymph nodes were enlarged (Fig. 1).

Then the patients underwent tracheotomy and resection of lesion, and the pathological examination was given. The pathologic diagnosis was the Castleman's disease.

\section{Discussion}

In recently, there were manly three viewpoints on the etiology of $\mathrm{CD}$. The first point was that the general inflammation of lymphatic drainage area or special inflammation of unknown cause; the second view was hamartoma of lymph node; the third was the tumor of cell anaplastic. But much more scholars support the first. They thought that the CD was found to be associated with human herpes virus 8 (HHV-8) and human immunodeficiency virus (HIV) infections [3], however, its causes remain unclear. The pathological grades were hyaline-vascular $\mathrm{CD}$, plasma cell $\mathrm{CD}$, and mixed CD [4]. Among them, the most is clear cell type, about 80-90\%; plasma cell type is rare about $8-9 \%$; the mixed is most rare.

The majority of clinical CD (UCD) were hyaline vascular type.Multicentric CD (MCD) also known as diffuse $\mathrm{CD}$, Most MCD belong to plasma cell type, The latter is more rare than the former [5], had the higher misdiagnosis rate [6], and more likely to die [7]. UCD general had no clinical symptoms, but it accompanied by fever, fatigue, weight loss, anemia and other systemic symptoms. It was found that by physical examination or routine chest X-ray. The peak age incidence of $\mathrm{CD}$ was between 30 and 40 years old, It's about 4 times more common in women than men. Preoperative diagnosis of UCD in the tracheal is very difficult, and surgical resection or excision is necessary. Complete resection is recommended for UCD because it is curative and has a 5 -year survival rate of $100 \%$. The most common site of UCD is mediastinum, it can also occur in the abdomen, neck, etc.

Patients with UCD are usually asymptomatic, and the diagnosis is achieved by imaging examination such as computed tomography (CT). The main imaging features of focal CD reviewed that was [8]: isolated, large and homogeneous soft tissue mass; and was no necrosis, hemorrhage and cystic change; the central part of the lesion may be accompanied by punctate or branching calcifications; in strengthening early stage (arterial phase) obviously even or uneven strengthening of mass, and tumor can be seen as persistent color enhancement by using contrast-enhanced CT. According to the predilection site and imaging features of CD [9-10], however, UCD needs to be differentiated from several blood rich tumors [11]: thymoma, carotid body tumor, ectopic pheochromocytoma, lymphoma, ectopic chemoreceptor tumors and lymph node metastases.

Althoogh the CD had certain characteristics of the image [12], but it was rare in clinical practice, so as to the misdiagnosis of preoperative still were. CD is a rare diease which can occur anywhere along the lymphatic chain. Previous literature reported that CD occurred in the orbital muscle cone [13-14], parapharyngeal space, neck, axillary, mediastinal, hilar area, small omentum, mesentery, etc. Even reported in the mediastinal cauased that the bronchial were compressed [15], however, it has never been reported in the literature that $\mathrm{CD}$ originated in the trachea. There was no primary and metastatic tumor in this case, according to CT's performance cannot get the right way to diagnosis, so the initial diagnosis we cannot considered to $\mathrm{CD}$. The preoprative differential diagnoses were thought tracheal adenoma, polyp of trachea, inflammatory myofibroblastic tumor of the trachea, and glomus tumor of the trachea. Finally, we rely on pathological diagnosis.

\section{Acknowledgements}

Study on distribution and metabolism of Gui Zhou Majiang blueberry anthocyanins in vivo Southeastern Guizhou J(2015)No.062 
Study on effects of blueberry extracts on the Protein Acetylation modification of Hepatocarcinoma Guizhou LH(2015)No.7433

Effects of blueberry on the proliferation, apoptosis and acetylation modifications of hepatocarcinoma Health and Family Planning Commission Guizhou(2015) NO.gzwjkj2015-1-037

Study on the effect of blueberry extract on the acetylation of liver fibrosis Southeastern Guizhou J (2015) No.081

\section{References}

[1] B. Castleman, L. Iversonl, and V.P. Menendez: Cancer, Vol. 9 (1956) No. 4, p.822.

[2] A.R. Gaba, R.S. Stein, and D.L. Sweet: Am J Clin Pathol, Vol. 69 (1978) No. 1, p.86.

[3] T. Johkoh, N.L. Müller, and K. Ichikado: Radiology, Vol.209 (1998) No. 2, p.477.

[4] D.M. Cronin, and R.A. Warnke: Adv Anat Pathol, Vol. 16 (2009) No. 4, p.236.

[5] E. Libson, S. Fields, and S. Strauss: Radiology, Vol. 166 (1988) No. 166, p.753.

[6] Q. Ding, J. Zhang, and L. Yang: Annals of Translational Medicine, Vol. 4 (2016) No.3, p.58.

[7] K. Gossios, C. Nikolaides, and M. Bai: European Radiology, Vol. 6(1996) No. 1, p.95.

[8] R. Madan, J.H. Chen, and B. Trotman-Dickenson: European Journal of Radiology, Vol. 81 (2012) No. 81, p.123.

[9] X.H. Jiang, H.M. Song, and Q.Y. Liu: European Journal of Radiology, Vol. 83 (2014) No. 11, p.2041.

[10] X. Zheng, K. Pan, and J. Cheng: Abdominal Radiology, Vol. 33 (2008) No. 4, p. 489.

[11]D.B. Koslin, L.L. Berland, and B.C. Sekar: Radiology, Vol. 160 (1986) No. 1, p.213.

[12] A.J. Hill, S.H. Tirumani, and M.H. Rosenthal: British Journal of Radiology, Vol. 88 (2015) No. 1049, p.20140670.

[13] A. Dong, H Dong, and C. Zuo: Clinical Nuclear Medicine, Vol. 39 (2014), No. 1, p.69.

[14] J.W. Brubaker, R.P. Harrie, and B.C. Patel: Ophthalmic Plastic \& Reconstructive Surgery, Vol. 27 (2011) No.2, p.e37.

[15] A. Haro, E. Kuramitsu, and Y. Fukuyama: International Journal of Surgery Case Reports, Vol. 25 (2016) No. C, p.44. 